05

\title{
Взаимодействие волн напряжений в твердых телах и сопровождающее электрическое поле
}

\author{
(C) Ю.К. Бивин
}

Институт проблем механики им. А.Ю. Ишлинского РАН, 119526 Москва, Россия

e-mail: ssb@post.com

(Поступило в Редакцию 10 марта 2016 г.)

Рассмотрено формирование электрически заряженных зон при взаимодействии волн деформаций в первоначально электрически нейтральном пластике. В эксперименте использован винипластовый стержень. Волны возбуждались ударом по концу стержня пулей в виде более короткого стержня из оргстекла или винипласта. Проведено сравнение экспериментальных результатов с предлагаемым математическим описанием.

Исследование механических динамических процессов с помощью регистрации сопровождающих электрических полей требует определенного представления о действующих напряжениях и деформациях. В случае поперечных колебаний балок или растянутых нитей при некотором расположении антенн возможно определить частоты и формы колебаний [1,2]. Это дает простой способ характеризовать не только динамические характеристики их движения, но и механические свойства их материала. Электрические заряды, возникающие во время движения тел в воздухе, позволяют с помощью установки по их траектории антенн оценить скорость и примерный размер тела вдоль вектора скорости [3]. Продольные волны в стержнях из диэлектрического материала, первоначально электрически нейтрального, создают электрически заряженные зоны, движущиеся вместе с волной. Знак заряда в зоне зависит от знака напряжений в волне. В отмеченных наблюдениях нарушение электрической нейтральности среды связано с ускорением ее частиц от действия сил. И чем больше это ускорение, тем больше плотность электрического заряда [4].

Как формируются электрические заряды при взаимодействии механических волн напряжений в среде? Этот вопрос рассматривается в случае движения продольных волн по цилиндрическому стержню со свободными плоскими концами. Винипластовый стержень диаметром $16 \mathrm{~mm}$ и длиной 1.1 или $1.5 \mathrm{~m}$ помещался вдоль линии стрельбы пневматической пушки калибром $10 \mathrm{~mm}$. Для возбуждения продольной волны деформаций выстрелы производились цилиндрическими стержнями диаметром $10 \mathrm{~mm}$ с плоскими торцами и длиной $40 \mathrm{~mm}$ из винипласта и $200 \mathrm{~mm}$ из оргстекла. Чтобы соударение со стержнем пули после вылета ее из ствола происходило с минимальным искажением условий плоского соударения, расстояние торца стержня от среза ствола составляло не более $0.3 \mathrm{~m}$. Скорость соударения ограничивалась $30-60 \mathrm{~m} / \mathrm{s}$, чтобы не происходило разрушения ни стержня, ни пули.

Волны напряжения при соударении стержней определяются на основании условий их прохождения через контактную плоскость. Они таковы $[5,6]$ :

$$
\begin{gathered}
F_{1}\left(\sigma_{1}+\sigma_{r}\right)=F_{2} \sigma_{t}, \\
v_{1}-v_{r}=v_{t},
\end{gathered}
$$

где $F_{1}, v_{1}, \sigma_{1}, \sigma_{r}$ - площадь поперечного сечения пули, ее скорость и напряжение в прямой и отраженной волнах. $F_{2}, v_{t}, \sigma_{t}$ - площадь поперечного сечения, скорость частиц и напряжение в стержне, возникающие при соударении.

На сновании связи между напряжением и скоростью частиц в волне $\sigma=\rho c \nu$, где $\rho, c$ - удельная плотность и скорость звука в среде, получаются следующие соотношения для напряжений в стержне и пуле:

$$
\begin{gathered}
\sigma_{t}=\frac{2 F_{1} c_{2} \rho_{2}}{F_{1} c_{1} \rho_{1}+F_{2} c_{2} \rho_{2}} \rho_{1} c_{1} v_{1}, \\
\sigma_{r}=\frac{F_{2} c_{2} \rho_{2}-F_{1} c_{1} \rho_{1}}{F_{1} c_{1} \rho_{1}+F_{2} c_{2} \rho_{2}} \rho_{1} c_{1} v_{1} .
\end{gathered}
$$

При используемых материалах и скоростях соударения $v_{1}$ в пределах $30-60 \mathrm{~m} / \mathrm{s}$ произведение $\rho_{1} c_{1} v_{1}$ при ударе винипластовой пулей равно $6.7 \cdot 10^{7}$ и $13.4 \cdot 10^{7}$ Ра. При ударе пулей из оргстекла $-7.35 \cdot 10^{7}$ и $14.7 \cdot 10^{7} \mathrm{~Pa}$. В выражениях для $\sigma_{t}$ и $\sigma_{r}$ эти цифры должны быть умножены на отношение параметров, учитывающих поперечные размеры и механические свойства соударяющихся тел. При ударе винипластовой пулей для $\sigma_{t}$ это дает значения напряжений в пределах $3.76 \cdot 10^{7}$ и $8.04 \cdot 10^{7} \mathrm{~Pa}$, а для $\sigma_{r}$ соответственно $2.93 \cdot 10^{7}$ и $5.87 \cdot 10^{7} \mathrm{~Pa}$. При использовании пули из оргстекла учет необходимой поправки для $\sigma_{t}$ приводит к $4.23 \cdot 10^{7}$ и $8.45 \cdot 10^{7} \mathrm{~Pa}$. А для $\sigma_{r}-$ к $2.94 \cdot 10^{7}$ и $5.88 \cdot 10^{7} \mathrm{~Pa}$.

Прочность винипласта на растяжение (4-6) $10^{7} \mathrm{~Pa}$, а на сжатие $(8-10) \cdot 10^{7} \mathrm{~Pa}$. У оргстекла прочность на растяжение $(6.5-7.8) \cdot 10^{7} \mathrm{~Pa}$. Эти данные характеризуют прочность при статических нагрузках. У пластических материалов в динамике обычно разрушающие напряжения гораздо выше. Поэтому в проведенных экспериментах при многократных ударах со скоростью 

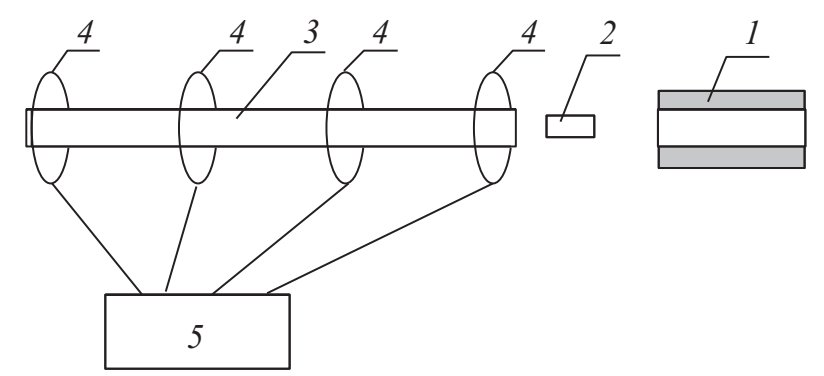

Pис. 1. Схема эксперимента. Пояснения обозначений в тексте.
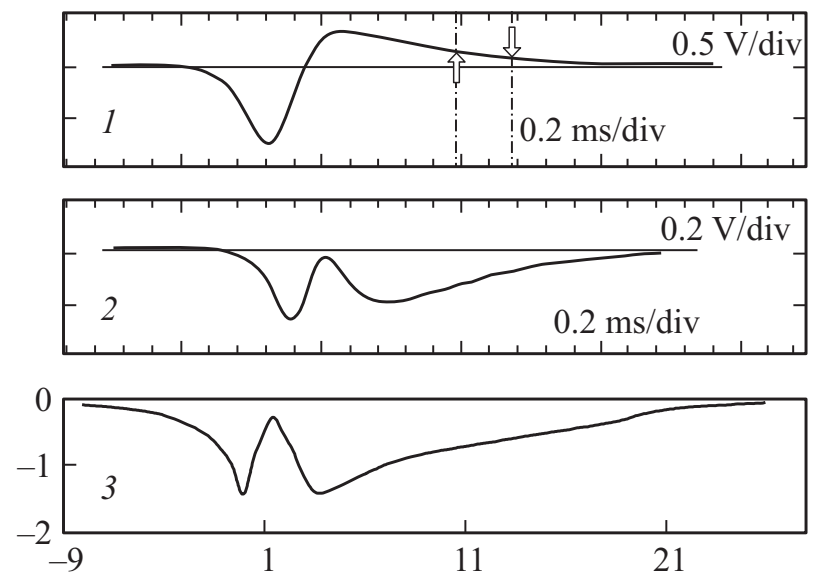

Рис. 2. Сравнение осциллограмм с антенн, установленных посредине 1 и плоскости свободного торца стержня 2.

$60 \mathrm{~m} / \mathrm{s}$ иногда происходило разрушение конца стержня в момент удара.

На рис. 1 показана схема эксперимента. Здесь $1-$ ствол пушки, 2 - пуля, 3 - стержень, 4 - антенны, 5 - осциллограф LeCroy. Стержень проходит сквозь кольцевые антенны. Запуск осциллографа происходит от сигнала, поступающего с антенны, установленной в плоскости торца стержня, по которому происходит удар.

На рис. 2 представлена осциллограмма, полученная при ударе винипластовой пулей по стержню длиной $1.1 \mathrm{~m} .1$ - сигнал с антенны, установленной в средней части стержня. В [4] показано, что в первоначально электрически нейтральном стержне при возбуждении в нем волны сжатия вместе с ней движется такой же длины отрицательно заряженная зона, а за ней - зона противоположного знака, несущая по абсолютной величине такой же заряд. Наиболее близко к реальным осциллограммам дается представление их в виде движения отрицательного заряда, распределенного с постоянной плотностью на участке длиной, равной длине импульса, и движущегося непосредственно за ним положительного заряда, распределенного с постоянной плотностью на участке в пять раз длиннее. 2 - сигнал с антенны, установленной в плоскости свободного конца стержня. Длина импульса сжатия $8 \mathrm{~cm}$. До начала отражения волны деформации от конца стержня осциллограммы 1 и 2 схожи.

Чтобы получить возможность интерпретировать отличительные особенности осциллограммы 2, опишем картину взаимодействия продольных волн деформации прямой и отраженной на свободном конце стержня [5]. Решение волнового уравнения удобно в нашем случае представить в виде

$$
u=F\left(c_{0} t+x\right)+f\left(c_{0} t-x\right),
$$

где функции $F$ и $f$ определяются начальными условиями. В нашем эксперименте при ударе пули в стержне вначале возникает волна напряжений сжатия, близкая к прямоугольной. Если начало координат поместить на свободном конце стержня и ось $x$ направить вдоль него, то $F$ описывает прямую волну. Поскольку $\partial u / \partial x=\sigma_{x} E$, то на свободном конце стержня должно выполняться равенство

$$
F^{\prime}\left(c_{0} t+x\right)-f^{\prime}\left(c_{0} t-x\right)=0 .
$$

То есть волна сжатия, проходя через свободный конец, вызывает появление волны растяжения.

На рис. 3 показаны отдельные этапы этого процесса. Цифрами 1-4 показано напряженное состояние и поляризация стержня в различные моменты. Цифрой 1 показан подход импульса сжатия, несущего отрицательный заряд, к свободному концу стержня (зона $A$ ). Зона $B$ - положительный заряд, сопровождающий импульс сжатия. Цифрой 2 отмечен момент начального этапа отражения, когда на свободном конце стержня появляется зона растягивающих напряжений, заряженная положительно. В зоне $C$ (свободный конец стержня) должен появиться отрицательный заряд и уравновесить новый положительный заряд. Зона сжимающих напряжений уменьшилась, а следовательно, уменьшился ее заряд, что приводит к уменьшению сопровождающего ее положительного заряда. В дальнейшем при расчетах предполагается соответствующее уменьшение погонной плотности заряда на этом участке. Цифрой 3 показана ситуация, когда длина отраженного импульса стала больше половины первоначальной длины прямого.

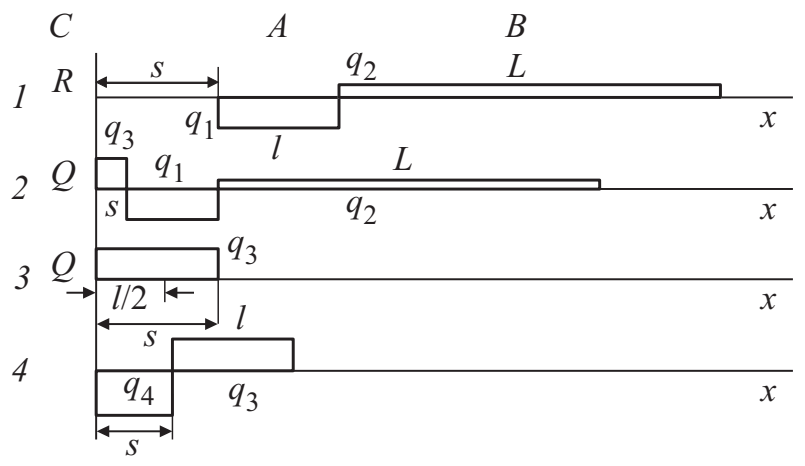

Рис. 3. Расчетная схема. 
В момент, когда длина отраженного импульса равна половине длины прямого, исчезает зона сжимающих напряжений, а одновременно с ней и сопровождающий ее положительный заряд. Зона растягивающих напряжений продолжает увеличиваться до длины начального импульса сжатия. За ней в зоне торца стержня возрастает соответственно отрицательный заряд. Цифрой 4 отмечено дальнейшее развитие процесса, в котором сопровождающая положительную отрицательно заряженная зона теперь имеет переменную плотность $q_{4}=(l / s) q_{1}$. Это продолжается до того момента, когда ее длина станет равной длине положительно заряженной зоны, показанной в момент 1. И в такой форме движение зарядов продолжается без изменений.

На первом этапе вычисляется напряженность поля при подходе импульса к концу стержня. Обозначения на всех этапах: $R$ - радиус антенны, $l$ - длина импульса сжатия, $q_{1}$ - плотность отрицательного заряда в зоне импульса сжатия, $L=5 l$ - длина положительного заряда, $\left|q_{2}\right|=(l / L)\left|q_{1}\right|$ - плотность положительного заряда на первом этапе, $s$ определяет положение зарядов на оси $x$, для которого вычисляется напряженность поля.

В этом случае напряженность $\varphi$ будет

$$
\varphi=-q_{1} \int_{s}^{s+l} \frac{d x}{\sqrt{R^{2}+x^{2}}}+0.2 q_{1} \int_{s+l}^{s+6 l} \frac{d x}{\sqrt{R^{2}+x^{2}}} .
$$

Отнеся к $R$, вводя обозначение $s / R=x$ и учитывая, что в эксперименте с использованием винипластовой пули $l / R=3$, получим

$$
\begin{aligned}
\varphi / q_{1}= & -\ln \frac{3+x+\sqrt{1+\left(3+x^{2}\right)}}{x+\sqrt{1+x^{2}}} \\
& +0.2 \ln \frac{18+x+\sqrt{1+(18+x)^{2}}}{3+x+\sqrt{1+x^{2}}} .
\end{aligned}
$$

Это справедливо пока $\infty \geq x \geq 0$. После начала отражения прямой волны вид расчетной схемы обозначен цифрой 2. Возникает волна растяжения длиной $s$, несущая положительный заряд плотностью $\left|q_{3}\right|=\left|q_{1}\right|$. Больцман предположил, что когда образец с внутренним трением испытывает ряд деформаций, действие каждой не зависит от других, и результирующее поведение можно вычислить путем простого сложения действий, которые имели бы такое действие, как если бы каждая деформация действовала одна. Это стало известно как принцип суперпозиции. Используя этот принцип здесь, необходимо предположить, что за волной растяжения на конце стержня появится заряд $Q=-q_{3} s$. Волна сжатия сокращается на $2 s$. И в связи с этим погонная плотность положительного заряда, ее сопровождающего, $\left|q_{2}\right|=\left|q_{1}\right|(l-2 s) / 5 l$.
Напряжение на антенне с учетом всех зарядов будет

$$
\begin{aligned}
\frac{\varphi}{\left|q_{1}\right|}= & -x+\ln \left(x+\sqrt{1+x^{2}}\right) \\
& -\ln \left(\frac{3-x+\sqrt{1+(3-x)^{2}}}{x+\sqrt{1+x^{2}}}\right) \\
& +\left(0.2-\frac{2 x}{15}\right) \ln \left(\frac{18-x+\sqrt{1+(18-x)^{2}}}{3-x+\sqrt{1+(3-x)^{2}}}\right)
\end{aligned}
$$

Вычисления производятся в пределах $0 \leq x \leq 1.5$. При $x=1.5$ исчезает отрицательно заряженный участок падающей волны сжатия и соответственно плотность сопровождающего положительного заряда становится равной нулю. Дальше расчет должен вестись с использованием первых двух членов вышеприведенного соотношения в пределах $1.5 \leq x \leq 3$. В этот момент заканчивается полное отражение прямой волны и в дальнейшем движении отраженной волны заряд на свободном конце стержня за ней, предполагаем, распределяется с постоянной плотностью. Расчетная схема показана под номером 3. Плотность $q_{4}$ отрицательного заряда меняется в соответствии с $\left|q_{4}\right|=\left|q_{1}\right|(l / s)$. В этом случае интегрирование дает

$$
\frac{\varphi}{\left|q_{1}\right|}=\ln \frac{3+x+\sqrt{1+(3+x)^{2}}}{x+\sqrt{1+x^{2}}}-\frac{3}{x} \ln \left(x+\sqrt{1+x^{2}}\right) .
$$

Вычисление ведется в пределах $0 \leq x \leq 15$. При $x \geq 15$ пара зарядов удаляется от свободного конца стержня в неизменном виде. Результат вычисления представлен на рис. 2 линией 3. Расчетная кривая отличается от осциллограммы 1 и отражает особенности осциллограммы 2.

Проведем сравнение результатов расчета и осциллограмм при установке антенн на расстоянии от свободного конца, равном половине и полной длины волны сжатия. При ударе по стержню пулей из оргстекла из-за разности скоростей движения волн деформаций длина волны сжатия в стержне будет $0.3 \mathrm{~m}$. Поэтому отношение $l / R=12$. Осциллограмма, полученная при установке антенны посредине стержня длиной $1.5 \mathrm{~m}$ показывает, что сопровождающий положительный заряд и в этом случае имеет длину примерно $0.4 \mathrm{~m}$ и тогда $L / R=16$. При таком значении получается и наиболее близкое к осциллограмме математическое представление напряженности поля. Сравнение представлено на рис. 4. Цифрой 1 отмечена осциллограмма, 2 - вычисление. При установке антенны на расстоянии половины длины импульса сжатия от свободного конца стержня и ударе пулей из винипласта напряженность поля изменяется, как показано осциллограммой 3. Результаты расчета представлены линией 4. При ударе винипластовой пулей и установке антенны на расстоянии длины импульса сжатия осциллограмма отмечена цифрой 5 , а расчет - 6 . Особенности осциллограмм отражаются в расчетных кривых, которые получены в предположении, что плотность отрицательного заряда постоянна в зоне первоначальной волны 

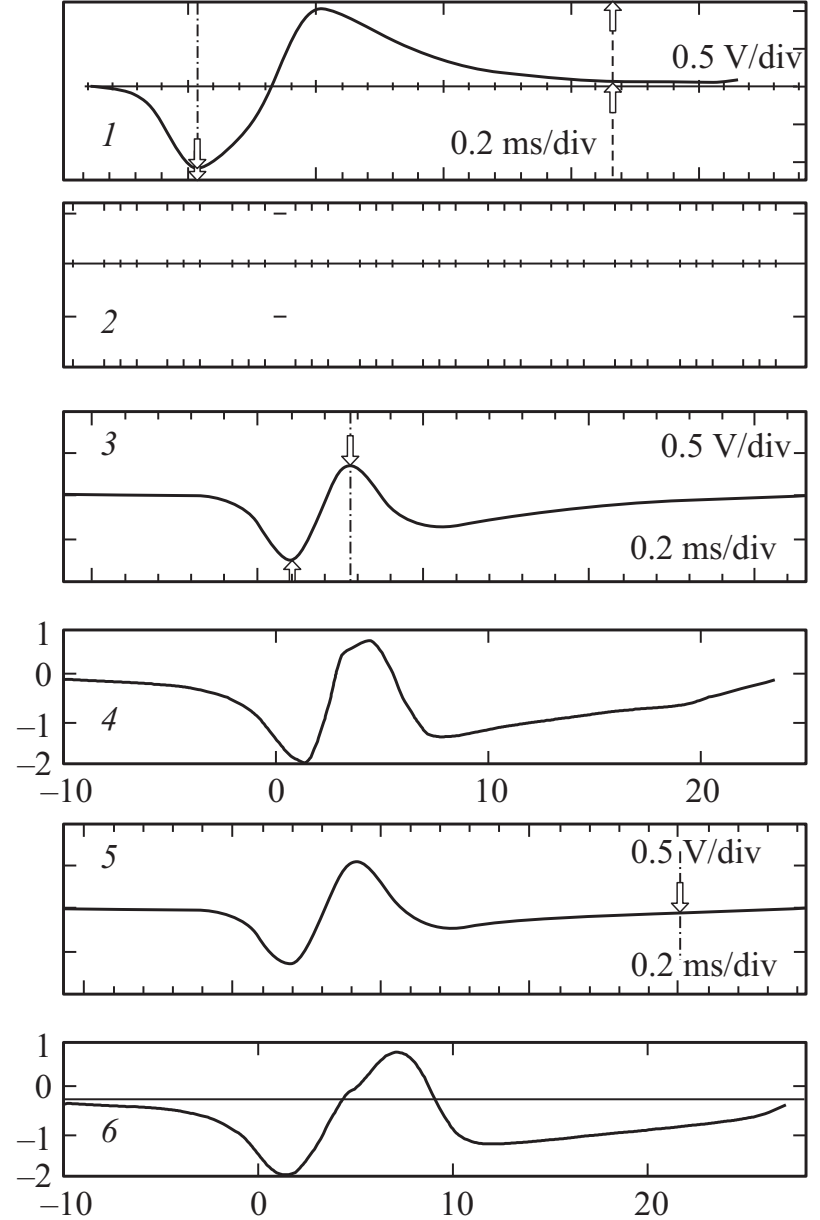

Рис. 4. Сравнение осциллограмм и расчета при установке антенн посредине стержня на расстоянии от свободного его конца, равном половине и полной длине волны сжатия.

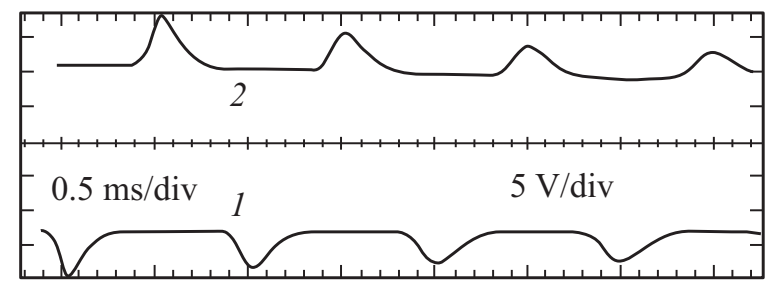

Рис. 5. Осциллограммы напряжений в плоскости торцов стержня, возникающие при отражении волн деформаций.

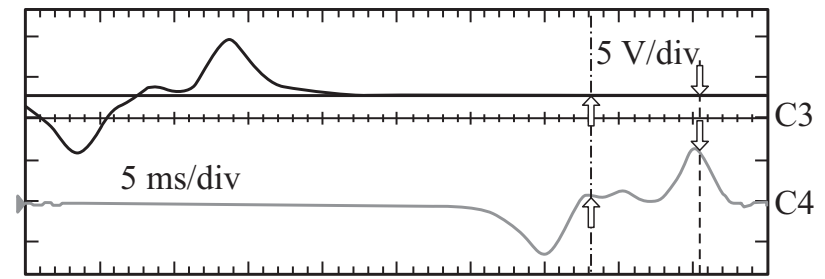

Рис. 6. Осциллограммы напряжений при пролете пульки из оргстекла через антенну. сжатия. Но сама волна сжатия не имеет строго прямоугольной формы. Причиной этому является не идеально плоское соударение, которое к тому же происходит в воздухе, и нелинейная зависимость деформации от напряжения у пластиков. Это приводит к тому, что расстояние между экстремумами, которые определяются длиной волны, на осциллограммах больше, чем в расчетах.

Осциллограммы антенн, установленных вблизи свободного конца стержня, получены в условиях, когда вблизи него устанавливалась многослойная металлическая заземленная сетка. При ее отсутствии антенна, установленная в плоскости свободного конца стержня или на некотором удалении от него, фиксирует напряженность поля, как показано на рис. 5. Здесь 1 сигнал с антенны в плоскости свободного конца, 2 - на конце, по которому произошел удар. Амплитуда сигнала на порядок выше, чем при прохождении импульса по стержню. Кроме того, форма соответствует проходу через антенну одного заряда. Импульсы на противоположных концах находятся в противофазе. Они вызваны взаимодействием торцов стержня с воздухом в процессе отражения соответствующих волн, на свободном сжатия, на противоположном - растяжения. Близкие по амплитуде и по форме напряжения возникают, когда через антенну пролетает пулька из оргстекла со скоростью, с которой происходил удар, как показано на рис. 6. Перед пулькой возникает заряд того же знака, что и вблизи свободного конца стержня, где в воздухе создается волна сжатия. Такое же соответствие имеет место и на противоположном конце стержня. На рисунке представлены показания антенн, установленных на некотором расстоянии, по которому определяется скорость распространения волны деформаций.

\section{Выводы}

1. Для описания результатов взаимодействия волн напряжения и сопровождающего электрического поля возможно использование принципа суперпозиции Больцмана.

2. Зарегистрировать взаимодействие зарядов, возникающих при отражении волн деформаций от свободных концов стержня, возможно при нейтрализации зарядов в воздухе, которые создаются движением торцов стержня.

Работа выполнена при финансовой поддержке гранта РФФИ № 14-08-00016.

\section{Список литературы}

[1] Бивин Ю.К. // ЖТФ. 2010. Т. 80. Вып. 6. С. 58-63.

[2] Бивин Ю.К. // ЖТФ. 2012. Т. 82. Вып. 6. С. 106-110.

[3] Бивин Ю.К. // ЖТФ. 2011. Т. 81. Вып. 10. С. 147-150.

[4] Бивин Ю.К. // ЖТФ. 2015. Т. 85. Вып. 6. С. 69-73.

[5] Кольский Г. Волны напряжений в твердых телах. М.: ИЛ, 1955. $192 \mathrm{c}$.

[6] Гольдсмит В. Удар. М.: Стройиздат, 1965. 448 с. 